\author{
Kouakou Léon Kobenan \\ UFR. CMS, Université Alassane Ouattara de Bouaké \\ $01 \mathrm{BpV} 18$ Bouaké 01, République de Côte d'Ivoire
}

\title{
La resémantisation contradictionnairique et ses implications dans Allah n'est pas obligé d'Ahmadou Kourouma
}

\begin{abstract}
There are atypical semantic neologisms in Allah n'est pas obligé by Ahmadou Kourouma, an Ivorian novelist. It is characterized by a redefinition of lexical items, which greatly contrasts with definitions provided by dictionaries. After a discussion of this phenomenon, called resémantisation contradictionnairique, the article shows Kourouma's clear intention to satirize African leaders and the French lexicographical institution, respectively accused of stirring up civil wars and giving partial and biased definitions.
\end{abstract}

Du fait qu'ils sont les répertoires des mots qui permettent de rendre compte de l'univers et de l'existence humaine dans tout leur fourmillement, les dictionnaires de langue sont perçus comme de respectables dépositaires des savoirs et des trésors culturels de l'humanité. C'est un mépris de ces précieux outils qu'on observe pourtant dans Allah n'est pas obligé, le quatrième roman d'Ahmadou Kourouma, un écrivain ivoirien (disparu en 2003), reconnu pour son écriture singulière caractérisée par l'intégration hardie au roman de formes relevant de l'Oralité africaine et de parlers marginaux. Le personnage principal de cette œuvre vilipende effectivement la 
lexicographie par sa manière excentrique de distordre les signifiés dictionnairiques de certains mots par des corrélats définitoires absolument incongrus. L'analyse suivante se propose de caractériser ce phénomène lexicographiquement dissonant, et aussi, d'en dégager les implications.

1. Une resémantisation originale et hétérodoxe

Birahima, l'auteur fictif d'Allah n'est pas obligé décide « de raconter [sa] vie» (11) à des destinataires d'origines et de conditions sociolinguistiques diverses.

1.1. Un projet de sémantisation singulier

Afin de rendre ses propos intelligibles, cet enfant-soldat se propose d'expliciter certaines lexies qu'il dénomme «gros mots » au moyen $\mathrm{du}$ Larousse, du Petit Robert, de l'Inventaire des particularités lexicales du français en Afrique noire et du dictionnaire Harrap's (11). C'est une démarche insolite car, généralement, les auteurs évoquent rarement les dictionnaires ayant servi dans leurs compositions. Toutefois, bien que destinée aux «Africains nègres noirs analphabètes » (11), la «traduction» de Birahima n'est pas bilingue, comme le montre la signification du syntagme « gros mots » (11). Loin de revêtir une quelconque consonance vulgaire, cette expression est un africanisme signifiant «mot savant, recherché » (Équipe IFA, 1988 : 173). Ainsi, même si le procédé ressemble à celui modélisé par les dictionnaires unilingues (qui associent un prédicat à une lexie donnée), il ne s'agit rien d'autre que d'un encodage consistant à passer d'un français soutenu à un français moyen, et non au petit-nègre. Car il définit simplement les mots qu'il juge difficiles au moyen desdits dictionnaires. Cependant, une huitaine de définitions, sur les dizaines que compte le texte, s'écartent nettement des définitions dictionnairiques. Celles-ci, quoique relativement peu nombreuses, ne sauraient constituer un banal épiphénomène, dans la mesure où elles sont la résultante $d^{\prime}$ ' " une prédication impertinente » (Klinkenberg, 1996 : 351) des différentes lexies. Ces (re)définitions sciemment dévoyées qui dédisent catégoriquement le projet initial de 
restituer le sens dictionnairique de certains mots apparaissent comme un écart qui requiert nécessairement une analyse. En effet, pour reprendre Olga Inkova, l'écart revêt « une signification dans l'énoncé [qu'il ne faut pas] considérer comme une simple erreur, mais comme un procédé » (Inkova, 2013 : 45). Avant de souligner les enjeux d'une telle déviance, il faut en relever la spécificité.

1.2. Une pratique lexicographique hétérodoxe

Le phénomène que nous appelons resémantisation contradictionnairique est une forme de néologie sémantique caractérisée par une redéfinition incohérente de mots prenant délibérément à rebours les définitions dictionnairiques. Il ressemble à l'idiosémie et à la néosémie ${ }^{1}$, sauf que celles-ci ne sont pas l'exutoire d'une verbigération étrange ou extravagante. Effectivement, comme le souligne Robert Hall (Gérard, 2010 : 14), l'idiosémie porte sur une allocation de sens uniquement liée aux aptitudes intellectuelles et cognitives réelles d'un individu. À l'opposé, la resémantisation contradictionnairique apparait, elle, comme un viol sémantique intentionnel. Dans l'étude, nous appliquerons le terme idiosème (emprunté à Hall) aux différents signifiés resémantisés incongrûment.

La néosémie contraste de la resémantisation contradictionnairique surtout au niveau de leur diffusion. Les signifiés néosémiques connaissent une vulgarisation appréciable après leur premier emploi. Selon Coralie Reutenauer, la néosémie est « une polysémie qui dure [et] qui nécessite de prendre en compte le facteur temps et la question de la répétition dans le temps » (Reutenauer, 2012: 41). À l'inverse, les signifiés relevant de la resémantisation contradictionnairique se présentent sous une seule occurrence et sont localisés exclusivement dans Allah n'est pas obligé. Quelques deux décennies après la parution de ce roman, ces nouveaux signifiés, à notre connaissance, n'ont pas été employés ailleurs. Et même si l'on se base sur le fait que de «manière générale, les néologismes mettent plusieurs dizaines

${ }^{1}$ Pour de plus amples informations sur ces notions, consulter : Gérard, 2010 ; Rastier et Valette, 2009 et Reutenauer, 2012. 
d'années avant d'entrer dans le dictionnaire » (Boussidan, 2009: 1), il est peu probable que ces idiosèmes soient un jour validés et diffusés, tant ils sont excentriques et rebelles à toutes les convenances lexicographiques et sémantiques.

Par ailleurs, à l'opposé du néosème qui devient une nouvelle acception de la lexie considérée, l'idiosème se caractérise par son sémantisme tranchant totalement avec ceux du/des corrélat(s) prédicatif(s) ancien(s). Si la lexie devient (plus) polysémique avec le néosème, qui est lui-même une nouvelle signification, l'idiosème contradictionnairique, en revanche, la racornit sémantiquement par (dé)vidage de son/ses sens ancien(s) à son seul profit. La lexie devient ainsi monosémique et se voit, en outre, affublée d'un sens généralement travesti et équivoque.

La resémantisation contradictionnairique s'observe à travers des gloses parenthétiques dont les significations fortement teintées de subjectivité s'écartent des signifiés avérés. Ce procédé la singularise encore plus car, bien que diverses, les gloses, les scholies et autres annotations métadiscursives s'inscrivent dans une tension constante vers un décryptage lexico-sémantique, historique ou socio-culturel du défini. Par cette volonté de désambigüisation maximale du référent, elles assument une fonction mathésique.

Il est vrai qu'il existe des ouvrages traitant de détournements sémantiques. Cependant, comme on l'observe dans Le dictionnaire détourné de Denis Saint-Amand, leurs titres extravagants révèlent d'emblée leur inscription dans une perspective satirique, humoristique ou dans un champ praxique extra-lexicographique. Par ailleurs, les possibles ambiguiités sur leurs orientations particulières sont assez tôt levées dans les incipit paratextuels ou dans l'introduction, qui alors, en indiquent les différentes singularités et/ou leurs motivations. Il importe à présent de comprendre comment Birahima a choisi d'empreindre si outrageusement le «code commun» (KerbratOrecchioni, $1980: 70$ ) qu'est la langue. 
2. La caractérisation de la resémantisation contradictionnairique L'étude prendra pour appui cinq cas, car les trois autres présentent avec ceux-ci des similitudes dans les différentes modalités d'appréciation et d'analyse. Les excentricités contradictionnairiques vont être dégagées au moyen de tableaux étoffant un schéma d'analyse de Matthieu-Colas (Mathieu-Colas, 2007: 1) par une prismatisation synoptique mettant en regard "l'énoncé de la signification générale» (Rastier et Valette, 2009 : 1) des lexies analysées et leurs correspondants resémantisés. Les idiosèmes seront abordés suivant la nomenclature classe/domaine. Un «domaine est un groupe de classes sémantiques (ou taxèmes) lié à une pratique sociale» (Rastier et Valette, 2009 : 1). L'analyse est axée autour de trois catégories d'idiosèmes.

2.1. La resémantisation par simple différenciation L'idiosème, bien que différent du signifié dictionnairique ne lui est pas antilogique, comme c'est généralement le cas.

(a) : «... (Parcours, c'est le trajet suivi par un petit toute sa courte vie sur terre, d'après mon Larousse) » (100).

Tableau 1. Idiosème différant simplement du signifié dictionnairique

\begin{tabular}{|c|c|c|c|}
\hline & Parcours & Classe & Domaine \\
\hline Dictionnaire & $\begin{array}{c}\text { Chemin qu'accomplit ou } \\
\text { que doit accomplir une } \\
\text { personne, un véhicule } \\
\text { pour aller d'un point à } \\
\text { un autre }\end{array}$ & Distance & Déplacement \\
\hline Birahima & $\begin{array}{c}\text { Trajet suivi par un petit } \\
\text { toute sa courte vie sur } \\
\text { terre }\end{array}$ & $\begin{array}{c}\text { Existence } \\
\text { tragiquement } \\
\text { brève des } \\
\text { enfants-soldats }\end{array}$ & Mort \\
\hline
\end{tabular}

Le mot parcours est étonnamment repris (192) avec l'une de ses acceptions conventionnelles : «Parcours signifie, d'après le Petit 
Robert, trajet suivi par quelqu'un ». Il est difficile de comprendre la raison de la signification détournée, alors que les deux occurrences sont toutes employées dans des «oraisons funèbres » $(93,100,192)$ célébrant des enfants-soldats tués. Également, on ne perçoit pas pourquoi la définition du Larousse seule est distordue. Quoi qu'il en soit, ce procédé traduit le caractère intentionnel de la déviance, car Birahima avait le loisir de revenir corriger le premier sens erroné. L'expression «d'après mon Larousse » est un leurre, car l'idiosème alloué à parcours s'applique uniquement et étonnamment à l'existence éphémère des enfants-soldats. Les idiosèmes que nous analysons dans les lignes suivantes s'inscrivent, eux, dans une resémantisation antinomique.

\subsection{La resémantisation par antilogie}

Ici, les idiosèmes entretiennent une relation antinomique avec les définitions du dictionnaire.

(b) : « [Le pédophile] se présenta, gentil et compatissant. (Compatissant, c'est-àdire faisant semblant de prendre part aux maux de Sarah)» $(95,96)$.

Tableau 2. Idiosème antilogique sur le plan taxémique

\begin{tabular}{|c|c|c|c|}
\hline & Compatissant & Classe & Domaine \\
\hline Dictionnaire & $\begin{array}{c}\text { Qui prend part aux } \\
\text { souffrances d'autrui; qui est } \\
\text { inspiré par la compassion }\end{array}$ & Sentiment & Psychologie \\
\hline Birahima & $\begin{array}{c}\text { Faire semblant de prendre } \\
\text { part aux maux de }\end{array}$ & Attitude & Psychologie \\
\hline
\end{tabular}

Même si elles relèvent du domaine Psychologie, les deux acceptions de compatissant sont cependant antinomiques au niveau taxémique, car le comportement malicieux, cruel et pervers du violeur de la petite 
orpheline Sarah se situe aux antipodes de la compassion. Dans l'exemple qui suit, l'antilogie est doublement accentuée.

(c) : «... (Ingérence humanitaire, c'est le droit qu'on donne à des États d'envoyer des soldats dans un autre État pour aller tuer des pauvres innocents chez eux, dans leur propre pays, dans leur propre village, dans leur propre case, sur leur propre natte)» (138).

Tableau 3. Idiosème antilogique aux niveaux taxémique et domanial

\begin{tabular}{|c|c|c|c|}
\hline Dictionnaire & $\begin{array}{c}\text { Ingérence humanitaire } \\
\text { internationale dans un pays afin } \\
\text { de secourir les populations } \\
\text { victimes de tueries, d'exactions } \\
\text { et d'autres violations des droits } \\
\text { de l'Homme }\end{array}$ & Altruisme & Dhilanthropie \\
\hline Birahima & $\begin{array}{c}\text { Devoir d'ingérence } \\
\text { d'envoyer des soldats dans un } \\
\text { autre État pour aller tuer des } \\
\text { pauvres innocents ... }\end{array}$ & Massacre & $\begin{array}{c}\text { Terrorisme } \\
\text { (institutionnalisé) }\end{array}$ \\
\hline
\end{tabular}

Avec les dictionnaires dont il dispose, Birahima aurait pu aisément fournir une définition claire d'ingérence humanitaire. Le sens resémantisé contraste tant avec la définition dictionnairique qu'avec les buts associés à cet interventionnisme. En effet, même s'il constitue un viol de la souveraineté nationale d'un État par d'autres États, l'ingérence humanitaire est sous-tendue par le principe qui détermine que, face à des violations massives des droits de l'Homme découlant d'une situation anomique dans un certain pays, la communauté internationale a le devoir de secourir les populations vulnérables, sous la forme d'une intervention militaro-humanitaire. Cependant avec l'acception singulière que Birahima confère à ce syntagme, on observe une redomanialisation. La redomanialisation est « un changement complet de classes sémantiques et de domaines » de départ (Rastier et 
Valette, 2009 : 7). Effectivement, le taxème Massacre et le domaine Terrorisme (institutionnalisé) dissonent de leurs correspondants respectifs Altruisme et Philanthropie.

2.3 La resémantisation par modification dans le champ catégoriel Les idiosèmes analysés dans cette partie redimensionnent le champ notionnel auquel appartient le signifié dictionnairique. Le premier lui adjoint une signification complémentaire tandis que le second en rétrécit grandement la portée.

(e) : «... (Dans mon Larousse, œcuménique signifie une messe dans laquelle ça parle de Jésus-Christ, de Mahomet et de Bouddha) » (57).

Tableau 4. Idiosème étoffant le champ notionnel du mot

\begin{tabular}{|c|c|c|c|}
\hline & Ecuménique & Classe & Domaine \\
\hline Dictionnaire & $\begin{array}{c}\text { Relatif au mouvement visant la } \\
\text { réunification des Églises } \\
\text { chrétiennes }\end{array}$ & $\begin{array}{c}\text { Unification } \\
\text { religieuse }\end{array}$ & Religion \\
\hline Birahima & $\begin{array}{c}\text { Une messe dans laquelle ça } \\
\text { parle de Jésus Christ, de } \\
\text { Mahomet et de Bouddha }\end{array}$ & $\begin{array}{c}\text { Unification } \\
\text { religieuse }\end{array}$ & Religion \\
\hline
\end{tabular}

Ici encore, la définition de Birahima est erronée, car eecuménique se rapporte à la doctrine ou au mouvement qui milite pour le rapprochement des Églises chrétiennes. Cette définition est singulière, car elle élargit considérablement le champ notionnel du mot à l'Islam et au Bouddhisme, par l'évocation de leurs fondateurs. À l'opposé, dans l'item suivant, l'idiosème remanie le contenu conceptuel de la lexie.

(f) : « ... (Coadjuteur signifie adjoint à un féticheur ») (205). 
Tableau 5. Éviction du signifié dictionnairique par l'idiosème

\begin{tabular}{|c|c|c|c|}
\hline & Coadjuteur & Classe & Domaine \\
\hline Dictionnaire & $\begin{array}{c}\text { Ecclésiastique nommé pour } \\
\text { aider un prélat à remplir ses } \\
\text { fonctions /Religieux servant } \\
\text { d'adjoint }\end{array}$ & Catholicisme & Religion \\
\hline Birahima & Adjoint à un féticheur & Animisme & Religion \\
\hline
\end{tabular}

Il y a d'abord la suppression du terme dictionnairique se rapportant au dignitaire religieux de la chrétienté, puis son éviction et sa commutation par féticheur. Par son cantonnement au seul animisme africain, le nouveau signifié bouleverse grandement le champ notionnel initial.

Tout écart étant sous-tendu par des motifs plus ou moins avoués, il importe de comprendre la portée des différentes redéfinitions extravagantes.

3. De l'idiosème à l' «idéosème » ou les enjeux idéologiques de la resémantisation contradictionnairique

Birahima qui est orphelin sillonne le Liberia et la Sierra Leone, deux pays déchirés par des guerres civiles à la recherche de sa tante Mahan. Il y devient un enfant-soldat dans différentes factions et est ainsi amené à tuer et à vivre des horreurs qui ne cesseront de le hanter. Impréparé à un tel bouleversement psychologique, il devient un traumatisé rebelle à toutes les figures de l'autorité. Cette psychogenèse permet de mieux cerner les causes de son verbe débridé et aigre qui s'exhale dans un langage démonté et coloré, qui, par moments, frise la néophasie. La néophasie est un mécanisme de défense constitué de néologismes qu'un psychopathe organise en un système lexical et syntaxique personnel ${ }^{2}$. Dans l'étude, ces

${ }^{2}$ À propos des conséquences de traumas tels ceux vécus par Birahima, voir Kobenan, $2013: 135-137$ 
néologismes correspondent aux idiosèmes. Ce sont des polarisateurs sémantiques qui charrient les opinions de Birahima. Étant idéologiquement marqués, ils se transmuent, pourrait-on dire, en idéosèmes. Ceux-ci, avant tout, anathématisent les dirigeants africains.

\subsection{La contemption des dirigeants africains}

La resémantisation inappropriée de parcours, compatissant et ingérence humanitaire constitue une satire des dirigeants africains dont l'égoïsme, conjoint à la désintégration de nombreuses familles, jette beaucoup d'enfants dans la rue. Dans ce vivier criminogène, certains deviennent la proie de malfaiteurs notoires. La redéfinition biaisée de compatissant traduit, par une froide ironie, la hideur de la fourbe machination par laquelle le pédophile parvint à violer Sara. D'autres encore, espérant trouver pitance et réconfort s'engagent volontairement dans les différentes factions, aux côtés de nombreux autres enrôlés de force. Drogués lors des combats et servant souvent de chair à canon, ils sont tués dans des conditions atroces. L'allocation de «courte vie » à parcours parait donc appropriée, car révélant mieux leur existence tragique et éphémère. Il faut, du reste, souligner que les distorsions sémantiques liées à compatissant et à parcours ont été faites dans le cadre d'un martyrologe qualifié d' "oraison funèbre ». Son but manifeste est de stigmatiser les massacres des enfants lors des guerres civiles. De fait au Liberia, selon l'UNICEF, «sur les 60.000 fighters, $10 \%$ [...] auraient moins de 15 ans, 30\% moins de 17 ans [tandis que d'autres] sources estiment la proportion de 12-17 ans à 70\% » (Weissman, 1996:8).

La resémantisation d'ingérence humanitaire traduit aussi l'écœurement de Birahima face aux carnages perpétrés contre les civils lors des opérations de l'ECOMOG (Economic Community of West African States Cease-fire Monitoring Group), la force d'interposition de la CEDEAO (151). Cet usage abusif et incontrôlé de la force armée et ses inéluctables effets collatéraux sur les populations désemparées, victimes, aussi bien des belligérants que des prétendus sauveurs, retraduit fictionnellement les exactions de l'ECOMOG pendant les guerres civiles libérienne et sierra léonaise. Il 
y eut, entre autres dérives, «le bombardement de la plantation de Firestone alors que [l'ECOMOG savait] que des civils [y] exploit[aient] du caoutchouc» (Bagayoko-Penone, 2004: 163). Hormis ces «regrettables bavures» (Wauthier, 2000: 1), le « commerce de biens pillés par des commandos locaux et des soldats de l'ECOMOG » (Atkinson, 1997 : 9) était la règle. Assimilée à une cohorte de pandours, la force dite d'interposition a vu le contenu de son acronyme [ECOMOG] être railleusement modifié en "Every Commodity Or Removable Object Gone"” (Bagayoko-Penone, 2004 : 161). « Outre, la satire politique, les extravagances sémantiques ciblent aussi l'institution lexicographique ».

3.2 Une entreprise de réforme lexicographique

Quand ils ne s'inscrivent pas dans une perspective purement badine, les néologismes sont généralement annonciateurs d'une revendication ou d'un parti pris linguistique, culturel ou idéologique. Similairement, les redéfinitions d'œecuménique et de coadjuteur se situent dans une entreprise de recadrage sémantico-culturel.

Le signifié dictionnairique d'œecuménique semble étroit pour Birahima. Ne référant qu'au mouvement visant l'unification de la seule chrétienté, il parait méconnaître les efforts de rapprochement entre les principaux courants religieux du monde, tel celui qui a réuni plus d'une soixantaine de responsables des grandes religions en 1986 à Assise. Comment donc nommer cet élan visant à l'unification de toutes les religions? Devant la déficience d'appellation de ce phénomène, Birahima a étendu la signification habituelle d'œecuménisme en associant à la chrétienté le Bouddhisme et l'Islam. $\mathrm{Du}$ reste, cet idiosème, bien que lexicographiquement nonconformiste, n'est pas si asémantique que cela, à cause du fait qu'effectivement, il cadre mieux avec la signification fédératrice originelle d'œecumenicus qui est « terre habitée, univers » (Rey, 2009, entrée æecuménique).

Bien que résultant d'un évincement du sens dictionnairique et non de l'étoffement du signifié conventionnel, la resémantisation de coadjuteur découle également d'un choix de recadrage sémantique. 
Puisque coadjuteur ne réfère qu'à l'adjoint du seul prélat de la chrétienté, et que par ailleurs, il n'existe aucun terme pour désigner l'adjoint de l'officiant animiste, Birahima a décidé de remplacer «prélat» par féticheur. Pourquoi a-t-il évité de juxtaposer sa définition à côté de celle du dictionnaire comme avec ocuménique ? C'est sans doute la traduction d'un emportement vindicatif, car, pour lui certainement, les sens étriqués d'œecuménique et de coadjuteur constituent un acte d'ostracisme culturel. Par ailleurs, non content d'altérer impudemment les définitions dictionnairiques, il pousse l'outrecuidance jusqu'à se rire des dictionnaires susmentionnés. À la page 74, il persifle Le Petit Robert : «Wallahé ! [Jurement de stupéfaction en malinké] Parfois le Petit Robert aussi se fout du monde ». Dans les propos suivants, ce sont Le Larousse et l'institution lexicographique française qui sont raillés à cause de l'usage de générale qu'il trouve aberrant: "Le général Baclay était une femme. (On devrait dire générale au féminin. Mais, d'après mon Larousse, "générale"' est réservé à la femme d'un général et jamais au général lui-même) » (110).

Il faut souligner que certaines descriptions et définitions de lexèmes impliquant l'Afrique reposent, aujourd'hui encore, sur des analyses ethnologiques largement tributaires des conceptions racistes de la période coloniale. Les altérations sémantiques liées à ocuménique et à coadjuteur symbolisent la dénonciation d'une vision du monde européocentrique de la lexicographie française. C'est cette idée que traduit sans doute le désaveu des deux dictionnaires par Birahima (au moins en ce qui concerne occuménique et coadjuteur). Cela est d'autant surprenant qu'au début de la rédaction de sa biographie, il proclamait sa foi infaillible dans les explicitations du Larousse et du Petit Robert. Cependant au fil des consultations, il se rend compte que ces dictionnaires qu'il considérait comme des vademecum infaillibles apparaissent lacunaires et partiaux.

\section{Conclusion}

L'analyse aura permis de mettre en relief un métalangage subversif caractérisé par un détournement sémantique corrosif qui satirise 
d'abord les dirigeants africains. Par des redéfinitions artificieuses de certaines lexies, Kourouma, sous la figure de Birahima, vitupère effectivement les politiques africains afin que cessent les effusions de sang provoquées par leur âpreté au gain. Il y a, encore, la raillerie de l'institution lexicographique française accusée d'instiller des définitions européistes parcellaires et partiales ; cela, par le biais de néologismes sémantiques dont l'incongruité déroute toutes les praxies lexicographiques. Cette attitude frondeuse traduit enfin l'invite que pendant les veilles lexicales (mises à jour des dictionnaires), les lexicographes devraient se sentir tenus autant à valider des néologismes qu'à revisiter les lexies anciennes, afin d'en affiner les définitions en les corrigeant ou en les actualisant au besoin. À l'occasion de cette nécessaire entreprise d'affinage, les lexies impliquant des considérations interculturelles devraient être analysées avec plus de méticulosité.

\section{Bibliographie}

Atkinson P. (1997) : L'économie de guerre au Libéria. Londres : Overseas Development Institute.

Bagayoko-Penone N. (2004) : Afrique : les stratégies françaises et américaines. Paris : L'Harmattan.

Boussidan A. et alii. (2009) : «La malbouffe : un cas de néologisme et de glissement sémantique fulgurants », Du thème au terme. Paris : INALCO, en ligne, http://12c2.isc.cnrs.fr/publications/files/TIA09.pdf (consulté le 3 avril 2014).

Équipe IFA. (1988) : Inventaire des particularités lexicales du français en Afrique noire. Paris : EDICEF/AUPEL.

Gérard C. (2010) : «L'individu et son langage : idiolecte, idiosémie, style », Texto, Vol. XV, n³, en ligne, http://www.revue-texto.net/index.php?id=2648 (consulté le 9 avril 2014).

Inkova O. (2013) : «Quand les erreurs sont-elles expressives ? », in : C. Chauvin, M. Kauffer (dir.), Écart et expressivité. La fonction expressive. Besançon : Presses Universitaires de Franche-Comté, pp.31-49.

Kerbrat-Orecchioni C. (1980): L'énonciation. De la subjectivité dans le langage. Paris : Armand Colin.

Klinkenberg J-M (1996) : Précis de sémiotique générale. Paris : De Boeck.

Kobenan Kouakou L. (2013) : «La double eschatologie de Maurice Bandaman ou la métaphorisation d'une ardente quête de justice sociale», Cahiers ERTA, $\mathrm{n}^{\circ} 4$, 
pp.125-138, en ligne,

http://cwf.ug.edu.pl/ojs/index.php/CE/issue/download/39/103.

Kourouma A. (2000) : Allah n'est pas obligé. Paris : Seuil.

Matthieu-Colas M. (2007) : «Domaines et classes sémantiques », Verbum, nº3/4, en ligne, https://halshs.archives-

ouvertes.fr/file/index/docid/433851/filename/Domaines_et_classes_semantiques.p df (consulté le 14 avril 2014).

Rastier F. et Valette M. (2009) : « De la polysémie à la néosémie », Texto, vol. XIV $\mathrm{n}^{\circ} 1$, en ligne, http://www.revuetexto.net/docannexe/file/2119/last_rastier_valette_polysemie.pdf (consulté le 04 avril 2014).

Reutenauer C. (2012) : Vers un traitement automatique de la néosémie, approche textuelle et statistique. Thèse de doctorat en Sciences du langage de l'Université de Lorraine.

Rey A. (2005) : Le Grand Robert de la langue française. Paris : SEJER.

Saint-Amand D. (2013) : Le dictionnaire détourné. Rennes : Presses Universitaires de Rennes.

Wauthier C. (2000) : «L'ONU, l'Afrique et ses diamants », Libération du $11 / 07 / 2000$.

Weissman F. (1996) : «Liberia, derrière le chaos, crise et interventions internationales », Relations Internationales et Stratégiques, $\mathrm{n}^{\circ} 24$. Paris : IRIS, en ligne, http://www.msf-crash.org/.../ce9c-fw-1996-liberia-derriere-le-chaos-criseset-i (consulté le 08 avril 2014). 